\title{
Existence results for classes of infinite semipositone problems
}

\author{
Jerome Goddard II', Eun Kyoung Lee ${ }^{2}$, Lakshmi Sankar ${ }^{3}$ and R Shivaji ${ }^{4 *}$
}

\footnotetext{
"Correspondence: shivaji@uncg.edu ${ }^{4}$ Department of Mathematics \& Statistics, University of North Carolina at Greensboro, Greensboro, NC 27412, USA

Full list of author information is available at the end of the article
}

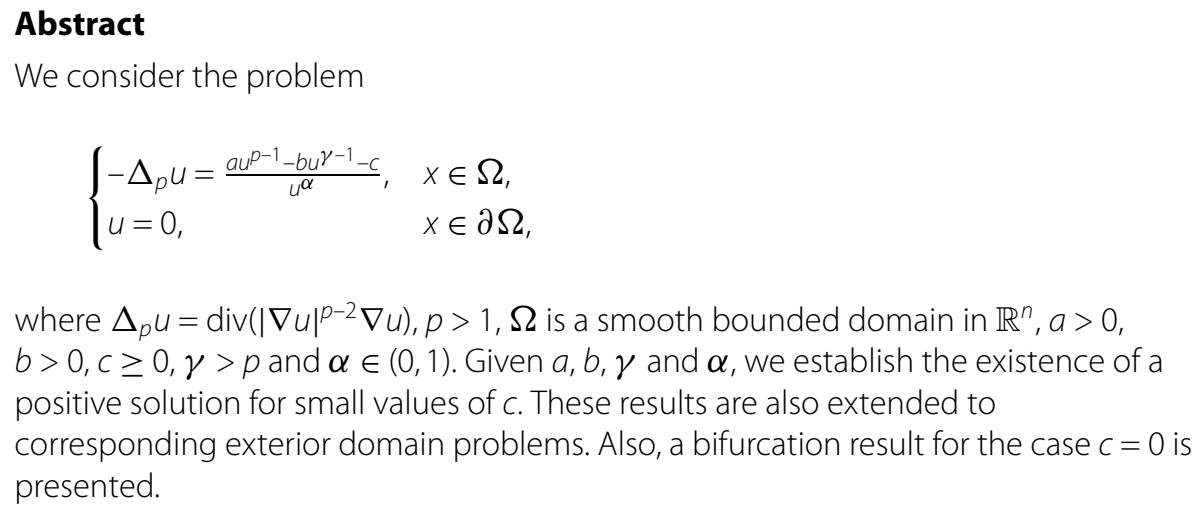

Abstract

We consider the problem

$$
\begin{cases}-\Delta_{p} u=\frac{a u^{p-1}-b{ }^{\gamma-1}-c}{u^{\alpha}}, & x \in \Omega, \\ u=0, & x \in \partial \Omega,\end{cases}
$$

where $\Delta_{p} u=\operatorname{div}\left(|\nabla u|^{p-2} \nabla u\right), p>1, \Omega$ is a smooth bounded domain in $\mathbb{R}^{n}, a>0$, $b>0, c \geq 0, \gamma>p$ and $\alpha \in(0,1)$. Given $a, b, \gamma$ and $\alpha$, we establish the existence of a positive solution for small values of $c$. These results are also extended to corresponding exterior domain problems. Also, a bifurcation result for the case $c=0$ is presented.

\section{Introduction}

Consider the nonsingular boundary value problem:

$$
\begin{cases}-\Delta u=a u-b u^{2}-\operatorname{ch}(x), & x \in \Omega, \\ u=0, & x \in \partial \Omega,\end{cases}
$$

where $\Omega$ is a smooth bounded domain in $\mathbb{R}^{n}, a>0, b>0, c \geq 0, \Delta u=\operatorname{div}(\nabla u)$ is the Laplacian of $u$ and $h: \bar{\Omega} \rightarrow R$ is a $C^{1}(\bar{\Omega})$ function satisfying $h(x) \geq 0$ for $x \in \Omega, h(x) \not \equiv 0$, $\max _{x \in \bar{\Omega}} h(x)=1$ and $h(x)=0$ for $x \in \partial \Omega$. Existence of positive solutions of problem (1) was studied in [1]. In particular, it was proved that given an $a>\lambda_{1}$ and $b>0$ there exists a $c^{*}(a, b, \Omega)>0$ such that for $c<c^{*}(1)$ has positive solutions. Here, $\lambda_{1}$ is the first eigenvalue of $-\Delta$ with Dirichlet boundary conditions. Nonexistence of a positive solution was also proved when $a \leq \lambda_{1}$. Later in [2], these results were extended to the case of the $p$-Laplacian operator, $\Delta_{p}$, where $\Delta_{p} u=\operatorname{div}\left(|\nabla u|^{p-2} \nabla u\right), p>1$. Boundary value problems of the form (1) are known as semipositone problems since the nonlinearity $f(s, x)=a s-b s^{2}-\operatorname{ch}(x)$ satisfies $f(0, x)<0$ for some $x \in \Omega$. See [3-9] for some existence results for semipositone problems.

In this paper, we study positive solutions to the singular boundary value problem:

$$
\begin{cases}-\Delta_{p} u=\frac{a u^{p-1}-b u^{\gamma-1}-c}{u^{\alpha}}, & x \in \Omega, \\ u=0, & x \in \partial \Omega,\end{cases}
$$

(c) 2013 Goddard II et al.; licensee Springer. This is an Open Access article distributed under the terms of the Creative Commons Attribution License (http://creativecommons.org/licenses/by/2.0), which permits unrestricted use, distribution, and reproduction in any medium, provided the original work is properly cited. 
where $\Delta_{p} u=\operatorname{div}\left(|\nabla u|^{p-2} \nabla u\right), p>1, \Omega$ is a smooth bounded domain in $\mathbb{R}^{n}, a>0, b>0$, $c \geq 0, \alpha \in(0,1), p>1$, and $\gamma>p$. In the literature, problems of the form (2) are referred to as infinite semipositone problems as the nonlinearity $f(s)=\frac{a s^{p-1}-b \gamma^{\gamma-1}-c}{s^{\alpha}}$ satisfies $\lim _{s \rightarrow 0^{+}} f(s)=-\infty$. One can refer to [10-14], and [15-17] for some recent existence results of infinite semipositone problems. We establish the following theorem.

Theorem 1.1 Given $a, b>0, \gamma>p$, and $\alpha \in(0,1)$, there exists a constant $c_{1}=c_{1}(a, b, \alpha, p, \gamma$, $\Omega)>0$ such that for $c<c_{1}$, (2) has a positive solution.

Remark 1.1 In the nonsingular case $(\alpha=0)$, positive solutions exist only when $a>\lambda_{1}$ (the principal eigenvalue) (see $[1,2])$. But in the singular case, we establish the existence of a positive solution for any given $a>0$.

Next, we study positive radial solutions to the problem:

$$
\begin{cases}-\Delta_{p} u=K(|x|)\left(\frac{a u^{p-1}-b u^{\gamma-1}-c}{u^{\alpha}}\right), & x \in \Omega, \\ u=0, & \text { if }|x|=r_{0}, \\ u \rightarrow 0, & \text { as }|x| \rightarrow \infty\end{cases}
$$

where $\Omega=\left\{x \in \mathbb{R}^{n}|| x \mid>r_{0}\right\}$ is an exterior domain, $n>p, a>0, b>0, c \geq 0, \alpha \in(0,1)$, $p>1, \gamma>p$ and $K:\left[r_{0}, \infty\right) \rightarrow(0, \infty)$ belongs to a class of continuous functions such that $\lim _{r \rightarrow \infty} K(r)=0$. By using the transformation: $r=|x|$ and $s=\left(\frac{r}{r_{0}}\right)^{\frac{-n+p}{p-1}}$, we reduce (3) to the following boundary value problem:

$$
\left\{\begin{array}{l}
-\left(\left|u^{\prime}\right|^{p-2} u^{\prime}\right)^{\prime}=h(s)\left(\frac{a u^{p-1}-b u^{\gamma-1}-c}{u^{\alpha}}\right), \quad 0<s<1, \\
u(0)=u(1)=0
\end{array}\right.
$$

where $h(s)=\left(\frac{p-1}{n-p}\right)^{p} r_{0}^{p} s^{\frac{-p(n-1)}{n-p}} K\left(r_{0} s^{\frac{-(p-1)}{n-p}}\right)$. We assume:

$\left(H_{1}\right) K \in C\left(\left[r_{0}, \infty\right),(0, \infty)\right)$ and satisfies $K(r)<\frac{1}{r^{n+\theta}}$ for $r \gg 1$, and for some $\theta$ such that $\left(\frac{n-p}{p-1}\right) \alpha<\theta<\frac{n-p}{p-1}$.

With the condition $\left(H_{1}\right), h$ satisfies:

there exists $\epsilon_{1}>0$ such that $h(s) \leq \frac{1}{s^{\rho}}$ for all $s \in\left(0, \epsilon_{1}\right)$,

$$
\text { where } \rho=\frac{n-p-\theta(p-1)}{n-p} \text {. }
$$

We note that if $\theta \geq \frac{n-p}{p-1}$ then $h(s)$ is nonsingular at 0 and $h \in C([0,1],(0, \infty))$. In this case, problem (4) can be studied using ideas in the proof of Theorem 1.1. Hence, our focus is on the case when $\theta<\frac{n-p}{p-1}$ in which, $h$ may be singular at 0 . Note that in this case $\hat{h}=$ $\inf _{s \in(0,1)} h(s)>0$.

Remark 1.2 Note that $\rho+\alpha<1$ since $\theta>\left(\frac{n-p}{p-1}\right) \alpha$.

We then establish the following theorem. 


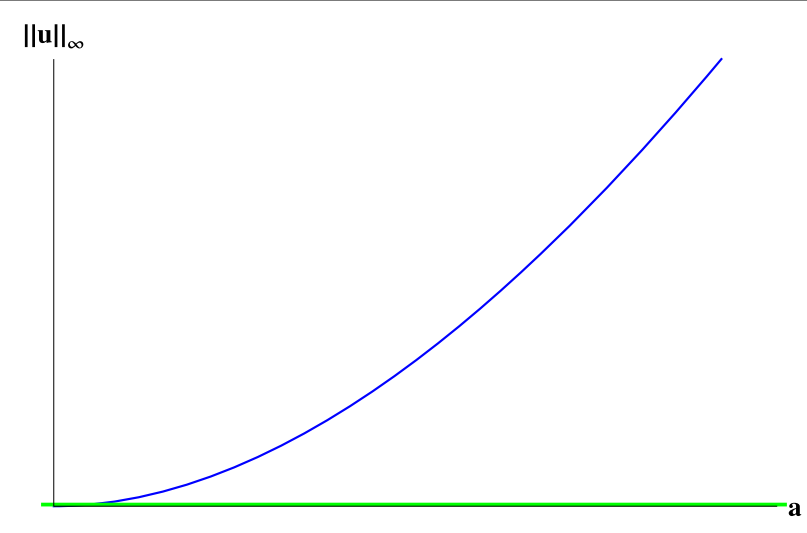

Figure 1 Bifurcation diagram, $a$ vs. $\|u\|_{\infty}$ for (6).

Theorem 1.2 Given $a, b>0, \gamma>p, \alpha \in(0,1)$, and assume $\left(H_{1}\right)$ holds. Then there exists $a$ constant $c_{2}=c_{2}(a, b, \alpha, p, \gamma)>0$ such that for $c<c_{2}$, (3) has a positive radial solution.

Finally, we prove a bifurcation result for the problem

$$
\begin{cases}-\Delta_{p} u=\frac{a u^{p-1}-b u^{\gamma-1}}{u^{\alpha}}, & x \in \Omega, \\ u=0, & \text { on } \partial \Omega,\end{cases}
$$

where $\Omega$ is a smooth bounded domain in $\mathbb{R}^{n}, a$ is a positive parameter, $b, \alpha>0, p>1+\alpha$ and $\gamma>p$. We prove the following.

Theorem 1.3 The boundary value problem (6) has a branch of positive solutions bifurcating from the trivial branch of solutions $(a, 0)$ at $(0,0)$ (as shown in Figure 1).

Our results are obtained via the method of sub-super solutions. By a subsolution of (2), we mean a function $\psi \in W^{1, p}(\Omega) \cap C(\bar{\Omega})$ that satisfies

$$
\begin{cases}\int_{\Omega}|\nabla \psi|^{p-2} \nabla \psi \cdot \nabla w d x \leq \int_{\Omega} \frac{a \psi^{p-1}-b \psi^{\gamma-1}-c}{\psi^{\alpha}} w d x, & \text { for every } w \in W \\ \psi>0, & \text { in } \Omega, \\ \psi=0, & \text { on } \partial \Omega\end{cases}
$$

and by a supersolution we mean a function $Z \in W^{1, p}(\Omega) \cap C(\bar{\Omega})$ that satisfies:

$$
\begin{cases}\int_{\Omega}|\nabla Z|^{p-2} \nabla Z \cdot \nabla w d x \geq \int_{\Omega} \frac{a Z^{p-1}-b Z^{\gamma-1}-c}{Z^{\alpha}} w d x, & \text { for every } w \in W \\ Z>0, & \text { in } \Omega, \\ Z=0, & \text { on } \partial \Omega,\end{cases}
$$

where $W=\left\{\xi \in C_{0}^{\infty}(\Omega) \mid \xi \geq 0\right.$ in $\left.\Omega\right\}$. The following lemma was established in [13].

Lemma 1.4 (see $[13,18])$ Let $\psi$ be a subsolution of $(2)$ and $Z$ be a supersolution of $(2)$ such that $\psi \leq Z$ in $\Omega$. Then (2) has a solution $u$ such that $\psi \leq u \leq Z$ in $\Omega$. 
Finding a positive subsolution, $\psi$, for such infinite semipositone problems is quite challenging since we need to construct $\psi$ in such a way that $\lim _{x \rightarrow \partial \Omega}-\Delta_{p} \psi=-\infty$ and $-\Delta_{p} \psi>0$ in a large part of the interior. In this paper, we achieve this by constructing subsolutions of the form $\psi=k \phi_{1}^{\beta}$, where $k$ is an appropriate positive constant, $\beta \in\left(1, \frac{p}{p-1}\right)$ and $\phi_{1}$ is the eigenfunction corresponding to the first eigenvalue of $-\Delta_{p} \phi=\lambda|\phi|^{p-2} \phi$ in $\Omega, \phi=0$ on $\partial \Omega$.

In Sections 2, 3, and 4, we provide proofs of our results. Section 5 is concerned with providing some exact bifurcation diagrams of positive solutions of $(2)$ when $\Omega=(0,1)$ and $p=2$.

\section{Proof of Theorem 1.1}

We first construct a subsolution. Consider the eigenvalue problem $-\Delta_{p} \phi=\lambda|\phi|^{p-2} \phi$ in $\Omega, \phi=0$ on $\partial \Omega$. Let $\phi_{1}$ be an eigenfunction corresponding to the first eigenvalue $\lambda_{1}$ such that $\phi_{1}>0$ and $\left\|\phi_{1}\right\|_{\infty}=1$. Also, let $\delta, m, \mu>0$ be such that $\left|\nabla \phi_{1}\right| \geq m$ in $\Omega_{\delta}$ and $\phi_{1} \geq \mu$ in $\Omega-\Omega_{\delta}$, where $\Omega_{\delta}=\{x \in \Omega \mid d(x, \partial \Omega) \leq \delta\}$. Let $\beta \in\left(1, \frac{p}{p-1+\alpha}\right)$ be fixed. Here, note that since $\alpha \in(0,1), \frac{p}{p-1+\alpha}>1$. Choose a $k>0$ such that $2 b k^{\gamma-p}+\beta^{p-1} \lambda_{1} k^{\alpha} \leq a$. Define $c_{1}=$ $\min \left\{k^{p-1+\alpha} \beta^{p-1}(\beta-1)(p-1) m^{p}, \frac{1}{2} k^{p-1} \mu^{\beta(p-1)}\left(a-\beta^{p-1} \lambda_{1} k^{\alpha}\right)\right\}$. Note that $c_{1}>0$ by the choice of $k$ and $\beta$. Let $\psi=k \phi_{1}^{\beta}$. Then

$$
-\Delta_{p} \psi=k^{p-1} \beta^{p-1} \lambda_{1} \phi_{1}^{\beta(p-1)}-k^{p-1} \beta^{p-1}(\beta-1)(p-1) \frac{\left|\nabla \phi_{1}\right|^{p}}{\phi_{1}^{p-\beta(p-1)}} .
$$

To prove $\psi$ is a subsolution, we need to establish:

$$
\begin{gathered}
k^{p-1} \beta^{p-1} \lambda_{1} \phi_{1}^{\beta(p-1)}-k^{p-1} \beta^{p-1}(\beta-1)(p-1) \frac{\left|\nabla \phi_{1}\right|^{p}}{\phi_{1}^{p-\beta(p-1)}} \\
\leq a k^{p-1-\alpha} \phi_{1}^{\beta(p-1-\alpha)}-b k^{\gamma-1-\alpha} \phi_{1}^{\beta(\gamma-1-\alpha)}-\frac{c}{k^{\alpha} \phi_{1}^{\alpha \beta}}
\end{gathered}
$$

in $\Omega$ if $c<c_{1}$. To achieve this, we split the term $k^{p-1} \beta^{p-1} \lambda_{1} \phi_{1}^{\beta(p-1)}$ into three, namely,

$$
\begin{aligned}
k^{p-1} \beta^{p-1} \lambda_{1} \phi_{1}^{\beta(p-1)}= & a k^{p-1-\alpha} \phi_{1}^{\beta(p-1-\alpha)}-\frac{1}{2} k^{p-1-\alpha} \phi_{1}^{\beta(p-1-\alpha)}\left(a-k^{\alpha} \phi_{1}^{\alpha \beta} \beta^{p-1} \lambda_{1}\right) \\
& -\frac{1}{2} k^{p-1-\alpha} \phi_{1}^{\beta(p-1-\alpha)}\left(a-k^{\alpha} \phi_{1}^{\alpha \beta} \beta^{p-1} \lambda_{1}\right) .
\end{aligned}
$$

Now to prove (7) holds in $\Omega$, it is enough to show the following three inequalities:

$$
\begin{aligned}
& -\frac{1}{2} k^{p-1-\alpha} \phi_{1}^{\beta(p-1-\alpha)}\left(a-k^{\alpha} \phi_{1}^{\alpha \beta} \beta^{p-1} \lambda_{1}\right) \leq-b k^{\gamma-1-\alpha} \phi_{1}^{\beta(\gamma-1-\alpha)}, \quad \text { in } \Omega, \\
& -\frac{1}{2} k^{p-1-\alpha} \phi_{1}^{\beta(p-1-\alpha)}\left(a-k^{\alpha} \phi_{1}^{\alpha \beta} \beta^{p-1} \lambda_{1}\right) \leq-\frac{c}{k^{\alpha} \phi_{1}^{\alpha \beta}}, \quad \text { in } \Omega-\Omega_{\delta}, \\
& -k^{p-1} \beta^{p-1}(\beta-1)(p-1) \frac{\left|\nabla \phi_{1}\right|^{p}}{\phi_{1}^{p-\beta(p-1)}} \leq-\frac{c}{k^{\alpha} \phi_{1}^{\alpha \beta}}, \quad \text { in } \Omega_{\delta} .
\end{aligned}
$$

From the choice of $k,-\left(a-\beta^{p-1} \lambda_{1} k^{\alpha}\right) \leq-2 b k^{\gamma-p}$, hence,

$$
\begin{aligned}
& -\frac{1}{2} k^{p-1-\alpha} \phi_{1}^{\beta(p-1-\alpha)}\left(a-k^{\alpha} \phi_{1}^{\alpha \beta} \beta^{p-1} \lambda_{1}\right) \leq-b k^{\gamma-1-\alpha} \phi_{1}^{\beta(p-1-\alpha)} \\
& \leq-b k^{\gamma-1-\alpha} \phi_{1}^{\beta(\gamma-1-\alpha)} .
\end{aligned}
$$


Using $\phi_{1} \geq \mu$ in $\Omega-\Omega_{\delta}$ and $c<\frac{1}{2} k^{p-1} \mu^{\beta(p-1)}\left(a-\beta^{p-1} \lambda_{1} k^{\alpha}\right)$

$$
\begin{aligned}
-\frac{1}{2} k^{p-1-\alpha} \phi_{1}^{\beta(p-1-\alpha)}\left(a-k^{\alpha} \phi_{1}^{\alpha \beta} \beta^{p-1} \lambda_{1}\right) & \leq \frac{-k^{p-1} \phi_{1}^{\beta(p-1)}\left(a-k^{\alpha} \lambda_{1} \beta^{p-1}\right)}{2 k^{\alpha} \phi_{1}^{\alpha \beta}} \\
& \leq \frac{-c}{k^{\alpha} \phi_{1}^{\alpha \beta}} .
\end{aligned}
$$

Finally, since $\left|\nabla \phi_{1}\right| \geq m$, in $\Omega_{\delta}$, and $c<k^{p-1+\alpha} \beta^{p-1}(\beta-1)(p-1) m^{p}$,

$$
\begin{aligned}
-k^{p-1} \beta^{p-1}(\beta-1)(p-1) \frac{\left|\nabla \phi_{1}\right|^{p}}{\phi_{1}^{p-\beta(p-1)}} & \leq \frac{-k^{p-1+\alpha} \beta^{p-1}(\beta-1)(p-1) m^{p}}{k^{\alpha} \phi_{1}^{\alpha \beta} \phi_{1}^{p-\beta(p-1)-\alpha \beta}} \\
& \leq \frac{-c}{k^{\alpha} \phi_{1}^{\alpha \beta} \phi_{1}^{p-\beta(p-1+\alpha)}} .
\end{aligned}
$$

Since $p-\beta(p-1+\alpha)>0$,

$$
-k^{p-1} \beta^{p-1}(\beta-1)(p-1) \frac{\left|\nabla \phi_{1}\right|^{p}}{\phi_{1}^{p-\beta(p-1)}} \leq \frac{-c}{k^{\alpha} \phi_{1}^{\alpha \beta}} .
$$

From (11), (12) and (13) we see that equation (7) holds in $\Omega$, if $c<c_{1}$. Next, we construct a supersolution. Let $e$ be the solution of $-\Delta_{p} e=1$ in $\Omega, e=0$ on $\partial \Omega$. Choose $\bar{M}>0$ such that $\frac{a u^{p-1}-b u^{\gamma-1}-c}{u^{\alpha}} \leq \bar{M}^{p-1} \forall u>0$ and $\bar{M} e \geq \psi$. Define $Z=\bar{M} e$. Then $Z$ is a supersolution of (2). Thus, Theorem 1.1 is proven.

\section{Proof of Theorem 1.2}

We begin the proof by constructing a subsolution. Consider

$$
\begin{aligned}
& -\left(\left|\phi^{\prime}\right|^{p-2} \phi^{\prime}\right)^{\prime}=\lambda|\phi|^{p-2} \phi, \quad t \in(0,1), \\
& \phi(0)=\phi(1)=0 .
\end{aligned}
$$

Let $\phi_{1}$ be an eigenfunction corresponding to the first eigenvalue of (14) such that $\phi_{1}>0$ and $\left\|\phi_{1}\right\|_{\infty}=1$. Then there exist $d_{1}>0$ such that $0<\phi_{1}(t) \leq d_{1} t(1-t)$ for $t \in(0,1)$. Also, let $\epsilon<\epsilon_{1}$ and $m, \mu>0$ be such that $\left|\phi_{1}^{\prime}\right| \geq m$ in $(0, \epsilon] \cup[1-\epsilon, 1)$ and $\phi_{1} \geq \mu$ in $(\epsilon, 1-\epsilon)$. Let $\beta \in\left(1, \frac{p-\rho}{p-1+\alpha}\right)$ be fixed and choose $k>0$ such that $2 b k^{\gamma-p}+\frac{\beta^{p-1} \lambda_{1} k^{\alpha}}{\hat{h}} \leq a$. Define $c_{2}=$ $\min \left\{\frac{k^{p-1+\alpha} \beta^{p-1}(\beta-1)(p-1) m^{p}}{d_{1}^{\rho}}, \frac{1}{2} k^{p-1} \mu^{\beta(p-1)}\left(a-\frac{\beta^{p-1} \lambda_{1} k^{\alpha}}{\hat{h}}\right)\right\}$. Then $c_{2}>0$ by the choice of $k$ and $\beta$. Let $\psi=k \phi_{1}^{\beta}$. This implies that:

$$
-\left(\left|\psi^{\prime}\right|^{p-2} \psi^{\prime}\right)^{\prime}=k^{p-1} \beta^{p-1} \lambda_{1} \phi_{1}^{\beta(p-1)}-k^{p-1} \beta^{p-1}(\beta-1)(p-1) \frac{\left|\phi_{1}^{\prime}\right|^{p}}{\phi_{1}^{p-\beta(p-1)}} .
$$

To prove $\psi$ is a subsolution, we need to establish:

$$
\begin{aligned}
& k^{p-1} \beta^{p-1} \lambda_{1} \phi_{1}^{\beta(p-1)}-k^{p-1} \beta^{p-1}(\beta-1)(p-1) \frac{\phi_{1}^{\prime p}}{\phi_{1}^{p-\beta(p-1)}} \\
& \quad \leq h(t)\left(a k^{p-1-\alpha} \phi_{1}^{\beta(p-1-\alpha)}-b k^{\gamma-1-\alpha} \phi_{1}^{\beta(\gamma-1-\alpha)}-\frac{c}{k^{\alpha} \phi_{1}^{\alpha \beta}}\right) .
\end{aligned}
$$


Here, we note that the term $k^{p-1} \beta^{p-1} \lambda_{1} \phi_{1}^{\beta(p-1)}=\frac{\hat{h} k^{p-1} \beta^{p-1} \lambda_{1} \phi_{1}^{\beta(p-1)}}{\hat{h}} \leq h(t)\left(a k^{p-1-\alpha} \phi_{1}^{\beta(p-1-\alpha)}\right.$ $\left.\frac{1}{2} k^{p-1-\alpha} \phi_{1}^{\beta(p-1-\alpha)}\left(a-\frac{k^{\alpha} \phi_{1}^{\alpha \beta} \beta^{p-1} \lambda_{1}}{\hat{h}}\right)-\frac{1}{2} k^{p-1-\alpha} \phi_{1}^{\beta(p-1-\alpha)}\left(a-\frac{k^{\alpha} \phi_{1}^{\alpha \beta} \beta^{p-1} \lambda_{1}}{\hat{h}}\right)\right)$, where $\hat{h}=\inf _{s \in(0,1)} h(s)$. Now to prove $(15)$ holds in $(0,1)$, it is enough to show the following three inequalities:

$$
\begin{aligned}
& -\frac{1}{2} k^{p-1-\alpha} \phi_{1}^{\beta(p-1-\alpha)}\left(a-\frac{k^{\alpha} \phi_{1}^{\alpha \beta} \beta^{p-1} \lambda_{1}}{\hat{h}}\right) \leq-b k^{\gamma-1-\alpha} \phi_{1}^{\beta(\gamma-1-\alpha)}, \quad \text { in }(0,1), \\
& -\frac{1}{2} k^{p-1-\alpha} \phi_{1}^{\beta(p-1-\alpha)}\left(a-\frac{k^{\alpha} \phi_{1}^{\alpha \beta} \beta^{p-1} \lambda_{1}}{\hat{h}}\right) \leq-\frac{c}{k^{\alpha} \phi_{1}^{\alpha \beta}}, \quad \text { in }(\epsilon, 1-\epsilon), \\
& -k^{p-1} \beta^{p-1}(\beta-1)(p-1) \frac{\left|\phi_{1}^{\prime}\right|^{p}}{\phi_{1}^{p-\beta(p-1)}} \leq-\frac{c h(t)}{k^{\alpha} \phi_{1}^{\alpha \beta}}, \quad \text { in }(0, \epsilon] \cup[1-\epsilon, 1) .
\end{aligned}
$$

From the choice of $k,-\left(a-\frac{\beta^{p-1} \lambda_{1} k^{\alpha}}{\hat{h}}\right) \leq-2 b k^{\gamma-p}$, hence,

$$
\begin{aligned}
-\frac{1}{2} k^{p-1-\alpha} \phi_{1}^{\beta(p-1-\alpha)}\left(a-\frac{k^{\alpha} \phi_{1}^{\alpha \beta} \beta^{p-1} \lambda_{1}}{\hat{h}}\right) & \leq-b k^{\gamma-1-\alpha} \phi_{1}^{\beta(p-1-\alpha)} \\
& \leq-b k^{\gamma-1-\alpha} \phi_{1}^{\beta(\gamma-1-\alpha)} .
\end{aligned}
$$

Using $\phi_{1} \geq \mu$ in $(\epsilon, 1-\epsilon)$ and $c<\frac{1}{2} k^{p-1} \mu^{\beta(p-1)}\left(a-\frac{\beta^{p-1} \lambda_{1} k^{\alpha}}{\hat{h}}\right)$

$$
\begin{aligned}
-\frac{1}{2} k^{p-1-\alpha} \phi_{1}^{\beta(p-1-\alpha)}\left(a-\frac{k^{\alpha} \phi_{1}^{\alpha \beta} \beta^{p-1} \lambda_{1}}{\hat{h}}\right) & \leq \frac{-k^{p-1} \phi_{1}^{\beta(p-1)}\left(a-\frac{k^{\alpha} \lambda_{1} \beta^{p-1}}{\hat{h}}\right)}{2 k^{\alpha} \phi_{1}^{\alpha \beta}} \\
& \leq \frac{-c}{k^{\alpha} \phi_{1}^{\alpha \beta}} .
\end{aligned}
$$

Next, we prove (18) holds in $(0, \epsilon]$. Since $\left|\phi_{1}^{\prime}\right| \geq m$, and $p-\beta(p-1)>\alpha \beta+\rho$

$$
\begin{aligned}
-k^{p-1} \beta^{p-1}(\beta-1)(p-1) \frac{\left|\phi_{1}^{\prime}\right|^{p}}{\phi_{1}^{p-\beta(p-1)}} & \leq \frac{-k^{p-1+\alpha} \beta^{p-1}(\beta-1)(p-1) m^{p}}{k^{\alpha} \phi_{1}^{\alpha \beta} \phi_{1}^{\rho}} \\
& \leq \frac{-k^{p-1+\alpha} \beta^{p-1}(\beta-1)(p-1) m^{p}}{k^{\alpha} \phi_{1}^{\alpha \beta} d_{1}^{\rho} t^{\rho}}
\end{aligned}
$$

Since $h(t) \leq \frac{1}{t^{\rho}}$ in $(0, \epsilon]$, and $c<\frac{k^{p-1+\alpha} \beta^{p-1}(\beta-1)(p-1) m^{p}}{d_{1}^{\rho}}$,

$$
-k^{p-1} \beta^{p-1}(\beta-1)(p-1) \frac{\left|\phi_{1}^{\prime}\right|^{p}}{\phi_{1}^{p-\beta(p-1)}} \leq \frac{-c h(t)}{k^{\alpha} \phi_{1}^{\alpha \beta}} .
$$

Proving (18) holds in $[1-\epsilon, 1)$ is straightforward since $h$ is not singular at $t=1$. Thus, from equations (19), (20) and (21), we see that (15) holds in $(0,1)$. Hence, $\psi$ is a subsolution. Let $Z=\bar{M} e$ where $e$ satisfies $-\left(\left|e^{\prime}\right|^{p-2} e^{\prime}\right)^{\prime}=h(t)$ in $(0,1), e(0)=e(1)=0$ and $\bar{M}$ is such that $\frac{a u^{p-1}-b u^{\gamma-1}-c}{u^{\alpha}} \leq \bar{M}^{p-1} \forall u>0$ and $\bar{M} e \geq \psi$. Then $Z$ is a supersolution of (4) and there exists a solution $u$ of (4) such that $u \in[\psi, Z]$. Thus, Theorem 1.2 is proven.

\section{Proof of Theorem 1.3}

We first prove (6) has a positive solution for every $a>0$. We begin by constructing a subsolution. Let $\phi_{1}$ be as in the proof of Theorem 1.1 (see Section 2). Let $\beta \in\left(1, \frac{p}{p-1}\right)$, and 
choose a $k>0$ such that $b k^{\gamma-p}+\beta^{p-1} \lambda_{1} k^{\alpha} \leq a$. Let $\psi=k \phi_{1}^{\beta}$. Then

$$
-\Delta_{p} \psi=k^{p-1} \beta^{p-1} \lambda_{1} \phi_{1}^{\beta(p-1)}-k^{p-1} \beta^{p-1}(\beta-1)(p-1) \frac{\left|\nabla \phi_{1}\right|^{p}}{\phi_{1}^{p-\beta(p-1)}}
$$

To prove $\psi$ is a subsolution, we will establish:

$$
k^{p-1} \beta^{p-1} \lambda_{1} \phi_{1}^{\beta(p-1)} \leq a k^{p-1-\alpha} \phi_{1}^{\beta(p-1-\alpha)}-b k^{\gamma-1-\alpha} \phi_{1}^{\beta(\gamma-1-\alpha)}
$$

in $\Omega$. To achieve this, we rewrite the term $k^{p-1} \beta^{p-1} \lambda_{1} \phi_{1}^{\beta(p-1)}$ as $k^{p-1} \beta^{p-1} \lambda_{1} \phi_{1}^{\beta(p-1)}=$ $a k^{p-1-\alpha} \phi_{1}^{\beta(p-1-\alpha)}-k^{p-1-\alpha} \phi_{1}^{\beta(p-1-\alpha)}\left(a-k^{\alpha} \phi_{1}^{\alpha \beta} \beta^{p-1} \lambda_{1}\right)$. Now to prove (22) holds in $\Omega$, it is enough to show $-k^{p-1-\alpha} \phi_{1}^{\beta(p-1-\alpha)}\left(a-k^{\alpha} \phi_{1}^{\alpha \beta} \beta^{p-1} \lambda_{1}\right) \leq-b k^{\gamma-1-\alpha} \phi_{1}^{\beta(\gamma-1-\alpha)}$. From the choice of $k,-\left(a-\beta^{p-1} \lambda_{1} k^{\alpha}\right) \leq-b k^{\gamma-p}$, hence,

$$
\begin{aligned}
-k^{p-1-\alpha} \phi_{1}^{\beta(p-1-\alpha)}\left(a-k^{\alpha} \phi_{1}^{\alpha \beta} \beta^{p-1} \lambda_{1}\right) & \leq-b k^{\gamma-1-\alpha} \phi_{1}^{\beta(p-1-\alpha)} \\
& \leq-b k^{\gamma-1-\alpha} \phi_{1}^{\beta(\gamma-1-\alpha)} .
\end{aligned}
$$

Thus, $\psi$ is a subsolution. It is easy to see that $Z=\left(\frac{a}{b}\right)^{\frac{1}{\gamma-p}}$ is a supersolution of (6). Since $k$, can be chosen small enough, $\psi \leq Z$. Thus, (6) has a positive solution for every $a>0$. Also, all positive solutions are bounded above by $Z$. Hence, when $a$ is close to 0 , every positive solution of (6) approaches 0 . Also, $u \equiv 0$ is a solution for every $a$. This implies we have a branch of positive solutions bifurcating from the trivial branch of solutions $(a, 0)$ at $(0,0)$.

\section{Numerical results}

Consider the boundary value problem

$$
\left\{\begin{array}{l}
-u^{\prime \prime}(x)=\frac{a u-b u^{2}-c}{u^{\alpha}}, \quad x \in(0,1) \\
u(0)=0=u(1)
\end{array}\right.
$$

where $a, b>0, c \geq 0$ and $\alpha \in(0,1)$. Using the quadrature method (see [19]), the bifurcation diagram of positive solutions of (23) is given by

$$
G(\rho, c)=\int_{0}^{\rho} \frac{d s}{\sqrt{[2(F(\rho)-F(s))]}}=\frac{1}{2}
$$

where $F(s):=\int_{0}^{s} f(t) d t$ where $f(t)=\frac{a t-b t^{2}-c}{t^{\alpha}}$ and $\rho=u\left(\frac{1}{2}\right)=\|u\|_{\infty}$. We plot the exact bifurcation diagram of positive solutions of (23) using Mathematica. Figure 2 shows bifurcation diagrams of positive solutions of (23) when $a=8\left(<\lambda_{1}\right)$ and $b=1$ for different values of $\alpha$.

Bifurcation diagrams of positive solutions of (23) when $a=15\left(>\lambda_{1}\right)$ and $b=1$ for different values of $\alpha$ is shown in Figure 3.

Finally, we provide the exact bifurcation diagram for (6) when $p=2$, and $\Omega=(0,1)$. Consider

$$
\left\{\begin{array}{l}
-u^{\prime \prime}(x)=\frac{a u-b u^{2}}{u^{\alpha}}, \quad x \in(0,1), \\
u(0)=0=u(1)
\end{array}\right.
$$




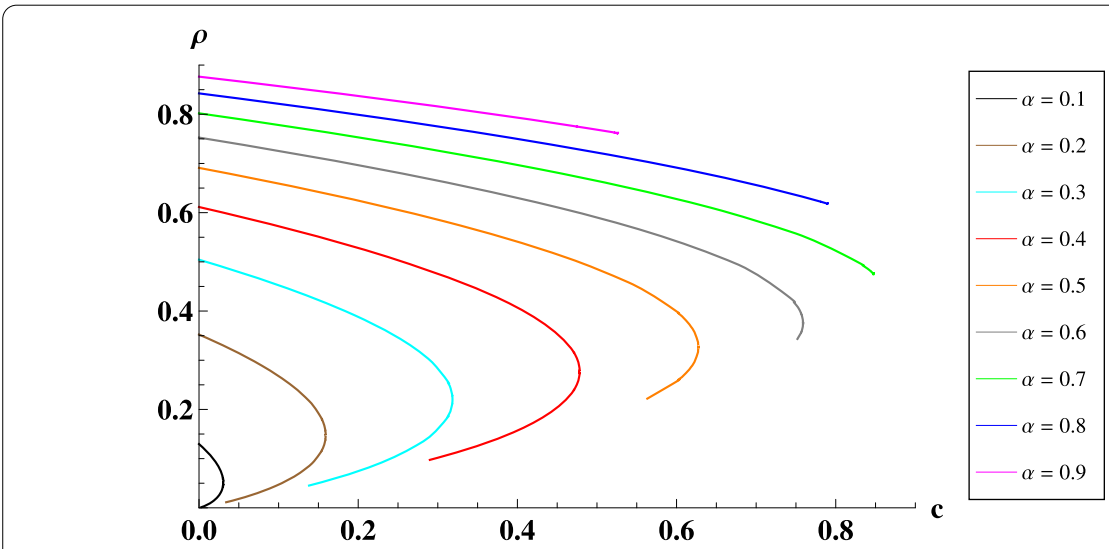

Figure 2 Bifurcation diagrams, $c$ vs. $\rho$ for (23) with $a=8, b=1$.
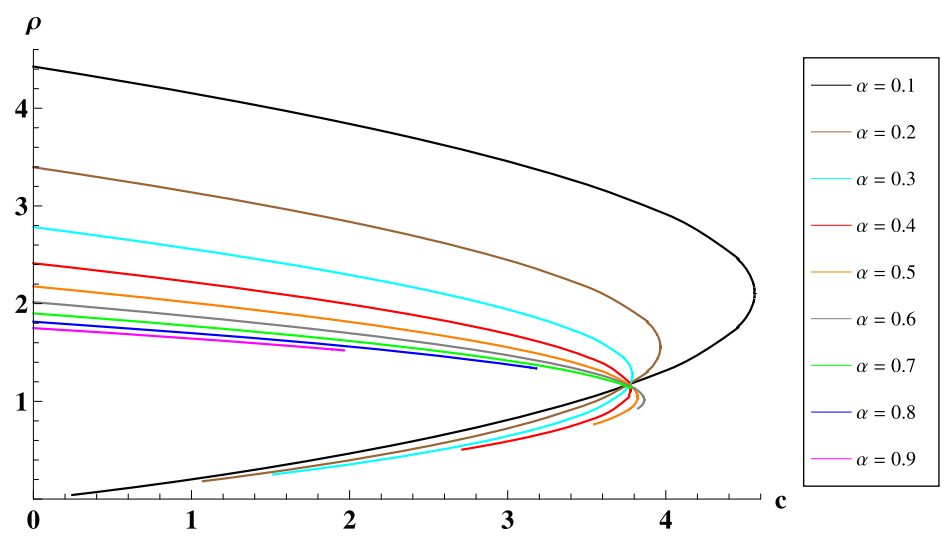

Figure 3 Bifurcation diagrams, $c$ vs. $\rho$ for (23) with $a=15, b=1$.

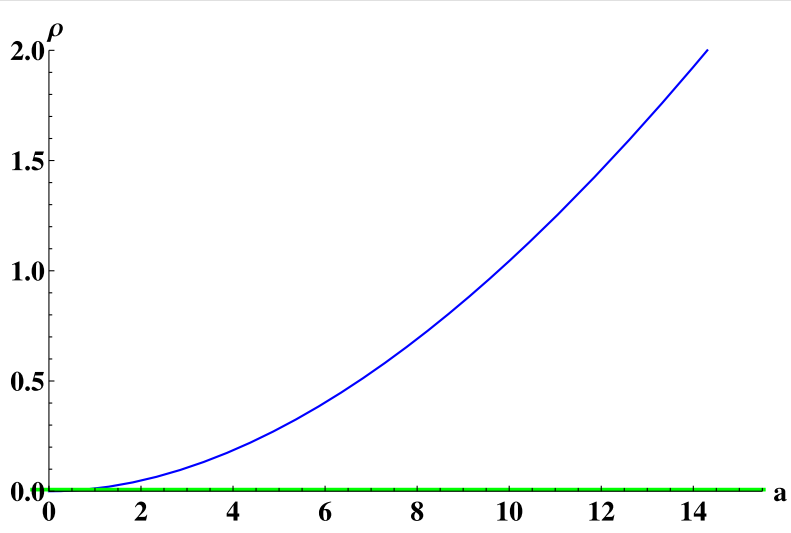

Figure 4 Bifurcation diagram, $a$ vs. $\rho$ for (25) with $\alpha=0.5, b=1$. 
where $a, b, \alpha>0$. The bifurcation diagram of positive solutions of (25) is given by

$$
\tilde{G}(\rho, a)=\int_{0}^{\rho} \frac{d s}{\sqrt{[2(\tilde{F}(\rho)-\tilde{F}(s))]}}=\frac{1}{2},
$$

where $\tilde{F}(s):=\int_{0}^{s} \tilde{f}(t) d t$ where $\tilde{f}(t)=\frac{a t-b t^{2}}{t^{\alpha}}$ and $\rho=u\left(\frac{1}{2}\right)=\|u\|_{\infty}$. The bifurcation diagram of positive solutions of (25) as well as the trivial solution branch are shown in Figure 4 when $\alpha=0.5$ and $b=1$.

\section{Competing interests}

The authors declare that they have no competing interests.

\section{Authors' contributions}

Equal contributions from all authors.

\section{Author details}

'Department of Mathematics, Auburn University Montgomery, Montgomery, AL 36124, USA. ${ }^{2}$ Department of Mathematics Education, Pusan National University, Busan, 609-735, Korea. ${ }^{3}$ Department of Mathematics \& Statistics, Mississippi State University, Mississippi State, MS 39762, USA. ${ }^{4}$ Department of Mathematics \& Statistics, University of North Carolina at Greensboro, Greensboro, NC 27412, USA.

\section{Acknowledgements}

EK Lee was supported by 2-year Research Grant of Pusan National University.

Received: 23 October 2012 Accepted: 5 April 2013 Published: 19 April 2013

\section{References}

1. Oruganti, S, Shi, J, Shivaji, R: Diffusive logistic equation with constant harvesting, I: steady states. Trans. Am. Math. Soc. 354(9), 3601-3619 (2002)

2. Oruganti, S, Shi, J, Shivaji, R: Logistic equation with the $p$-Laplacian and constant yield harvesting. Abstr. Appl. Anal. 9 723-727 (2004)

3. Ambrosetti, A, Arcoya, D, Biffoni, B: Positive solutions for some semipositone problems via bifurcation theory. Differ Integral Equ. 7, 655-663 (1994)

4. Anuradha, V, Hai, DD, Shivaji, R: Existence results for superlinear semipositone boundary value problems. Proc. Am. Math. Soc. 124(3), 757-763 (1996)

5. Arcoya, D, Zertiti, A: Existence and nonexistence of radially symmetric nonnegative solutions for a class of semipositone problems in an annulus. Rend. Mat. Appl. 14, 625-646 (1994)

6. Castro, A, Garner, JB, Shivaji, R: Existence results for classes of sublinear semipositone problems. Results Math. 23, 214-220 (1993)

7. Castro, A, Shivaji, R: Nonnegative solutions for a class of nonpositone problems. Proc. R. Soc. Edinb. 108(A), 291-302 (1998)

8. Castro, A, Shivaji, R: Nonnegative solutions for a class of radially symmetric nonpositone problems. Proc. Am. Math. Soc. 106(3), 735-740 (1989)

9. Castro, A, Shivaji, R: Positive solutions for a concave semipositone Dirichlet problem. Nonlinear Anal. 31, 91-98 (1998)

10. Ghergu, M, Radulescu, V: Sublinear singular elliptic problems with two parameters. J. Differ. Equ. 195, $520-536$ (2003)

11. Hai, DD, Sankar, L, Shivaji, R: Infinite semipositone problems with asymptotically linear growth forcing terms. Differ. Integral Equ. 25(11-12), 1175-1188 (2012)

12. Hernandez, J, Mancebo, FJ, Vega, JM: Positive solutions for singular nonlinear elliptic equations. Proc. R. Soc. Edinb. 137A, 41-62 (2007)

13. Lee, E, Shivaji, R, Ye, J: Classes of infinite semipositone systems. Proc. R. Soc. Edinb. 139(A), 853-865 (2009)

14. Lee, E, Shivaji, R, Ye, J: Positive solutions for elliptic equations involving nonlinearities with falling zeros. Appl. Math. Lett. 22, 846-851 (2009)

15. Ramaswamy, M, Shivaji, R, Ye, J: Positive solutions for a class of infinite semipositone problems. Differ. Integral Equ. 20(11), 1423-1433 (2007)

16. Shi, J, Yao, M: On a singular nonlinear semilinear elliptic problem. Proc. R. Soc. Edinb. 128A, 1389-1401 (1998)

17. Zhang, Z: On a Dirichlet problem with a singular nonlinearity. J. Math. Anal. Appl. 194, 103-113 (1995)

18. Cui, S: Existence and nonexistence of positive solutions for singular semilinear elliptic boundary value problems. Nonlinear Anal. 41, 149-176 (2000)

19. Laetsch, T: The number of solutions of a nonlinear two point boundary value problem. Indiana Univ. Math. J. 20, 1-13 (1970)

doi:10.1186/1687-2770-2013-97

Cite this article as: Goddard II et al.: Existence results for classes of infinite semipositone problems. Boundary Value Problems 2013 2013:97. 